\title{
ON THE QUASIUNIQUENESS OF SOLUTIONS OF DEGENERATE EQUATIONS IN HILBERT SPACE
}

\section{VLADIMIR SCHUCHMAN}

Departamento de Matheriaticas del Centro de Investigacion $y$ de

Estudios Avanzados del I.P.N. Apartado Pestal 14-740

Mexico, D.F. CP 07000-Mexico

(Recelved December 22, 1981, and in revised form Ajpril 15, 1982)

ABSTRACT. In this paper, we study the quasiuniciueness (i.e., $f_{1} \doteq f_{2}$ if $f_{1}-f_{2}$ is flat, the function: $f(t)$ being called flat if, for any $k>0, t^{-k} f(t) \rightarrow 0$ as $t \rightarrow 0)$ for crdinary differential ecuations in Hilbert space. The case of inequalities is studied, too.

The most important result of this paper is this:

THEOREM 3. Let $B(t)$ be a linear operator with domair $D_{B}$ and $B(t)=B_{1}(t)+$ $B_{2}(t)$ where $\left(B_{1}(t) x, x\right)$ is real and $\operatorname{Re}\left(E_{2}(t) x, x\right)=0$ for any $x \in D_{B}$. Let for any $x \in D_{B}$ the followirg estimate hold:

$$
\begin{array}{r}
\left\|B_{1} x-\frac{\left(B_{1} x, x\right)}{(x, x)} x\right\|^{2}+\operatorname{Re}\left(B_{1} x, B_{2} x\right)+t\left(B_{1}(t) x, x\right) \geq-C t\left[\mid\left(B_{1}(t) x, x\right) \|+(x, x)\right] \\
\text { with } C \geq 0 .
\end{array}
$$

If $u(t)$ is a smooth flat solution of the following inequality in the interval $t \in I=(0,1]$.

$$
\left\|t \frac{d u}{d t}-B(t) u\right\| \leq t_{\phi}(t)\|u(t)\|
$$

with non-negative continuous function $\phi(t)$, then $u(t) \equiv 0$ in $I$. One example with self-adjoint $B(t)$ is given, too.

KEY WORDS AND PHRASES. Degenerate equations, differential equations in Hilbert space, quasiuniqueness, flat solutions.

1980 MATHEMATICS SUBJECT CLASSIFICATION CODE. 34G10, 35J70, 35K22, 35K65, 35R45.

0. IRTRODUCTION.

In this paper we study the quasiuniqueness of solutions of the abstract equation of the form

$$
t \frac{d u}{d t}=B(t) u, t \quad I=[0, T], \quad 0<T<+\infty
$$

Here $B(t)$ is an unbounded non-symmetric operator in Hilbert space. The quasiuniqueness of solutions of a somewhat more general problem of the form

$$
\left\|t \frac{d u}{d t}-B(t) u(t)\right\| \leq t \phi(t)\|u(t)\|
$$


is stucied too. Here $B(t)$ is cif the same tiple $a s$ in $(0.1)$, aric $\phi(t)$ is a continuous non-regative function ir the interva? $!$.

Recall that by quasiuniqueress we meall liriqueness in the class of functions that differ by flat functions. We say that the firiction $u(t)$ is i flat furction if

$$
\forall k>0, t^{-k} u(t) \underset{t \rightarrow 0}{\longrightarrow} 0 \text {. }
$$

In Section 1, we study the simplest model, which is further cleveloped in Section 2. In Section 3 the main theorems are ubtained: Thecrem 3 for the problem (0.2) and Theorem 4 for the problem $(0.1)$. Our conditions of quasiuniqueness cerieralize the curresponding conditioris of [1] (we do not present, the analog of Theorem :-1 of [1] since it is trivial). Theorem 2 of section 2 corresponds to Theorem $1-3$ of $[1]$ and generalizes Theorem 1-2 of the same paper. Our Theorems 3, 4 of Section 3 are a further generalization of Theorem 2, section 2 as well as of Theorems 1-2, 1-3 of [1]. Section 4 is devoted to reriarks about previous sections. We point out that in the paper we used methods different from those of Alirihac-Baouendi in [1].

Problems $(0.1),(0.2)$ and those which can be reduced to them were recently studied by a number of authors (see [1-6]). Thus in [4] an example of a particular equation which could be reduced to the form $(0.1)$, where $B(t)=B(0)$ is selfadjoint, was considered, and the quasiuniqueness was proved for it. Further in [2] and [3], equation (0.1) was studied for $B(t)=B(0)+t B_{1}(t)$ with $E(0)$ bounded (Fuchs-type equation). In the paper [5], the quasiunicueness was proved for a certain class of elliptic operators with a degeneration in a single point. Conditicrs which are difficult to verify were imposed, but a simple class of elliptic operators satisfying them was indicated. In our paper [6] elliptic equations with a possible degeneration on a hyperplane or in a single point are studied. In [6], the quasiuniqueness was proved for $(0.1)-(0.2)$ with self-adjoint operator $B(t)$.

Methods employed here were first used by Agmon and Nirenberg ([7], [8]) for studying the Cauchy problem in the non-degenerate case.

\section{MODEL CASE.}

Let $H$ be a Hilbert space with scalar product $(\cdot, \cdot)$ and norm $\|\cdot\|$, I is the interval $[0, T]$ with $0<T<+\infty, \phi(t)$ a continuous non-negative function on $I$, $u(t) \in C^{1}(I, H), A$, a linear operator in $H$, with domain $D_{A}$ and $A=A_{1}+A_{2}$, $A_{1}^{*}=A_{1}$ the self-adjoint part of $A$, and $A_{2}^{*}=-A_{2}$ the anti-self-adjoint part of $A$. We shall assume that $u(t) \in D_{A}$ and that $A u(t) \in C(I, H)$. Set $D=t \frac{\partial}{\partial t}$.

THEOREM 1. Let $u(t)$ be a solution of the inequality

$$
\|D u(t)-A u(t)\| \leq t \phi(t)\|u(t)\| \text {. }
$$

We suppose that all the conditions introduced above hold and that commutator $\left[A_{1}, A_{2}\right]=0$. Let $u(t)$ be a flat function (i.e., $\forall k>0, t^{-k} u(t) \underset{t \rightarrow 0}{\longrightarrow} 0$ ). Then $u(t) \equiv 0$ in $I$.

PROOF. Let

$$
\begin{aligned}
& q(t)=(u(t), u(t)) \\
& f(t)=\operatorname{Du}(t)-A u(t),
\end{aligned}
$$

and let $\left(t_{1}, t_{0}\right]$ be a subinterval of $I$ such that $q(t)>0$ for $t_{1}<t \leq t_{0}$,

$$
\psi(t)=2 \operatorname{Re}(f(t), u(t)) / q(t)
$$




$$
\begin{aligned}
& s(t)=\exp -\int_{0}^{t} \frac{\psi(\tau)}{\tau} d \tau \\
& s(t)=\log [q(t) \cdot s(t) . .
\end{aligned}
$$

LEMMA 1.1. Suppose that all the corditions of Theorem 1 and (1.2)-(1.6) hold. Then $\ell(t)$ is twice differentiable and satisfies the followira second-order differential irecuality in the interval $\left(t_{1}, t_{0}\right]$ :

$$
D^{2} x(t)+2 t^{2} \phi^{2}(t) \geq 0 \text {. }
$$

PRO0F. Fron (1.6) we have

$$
\dot{t} \dot{s}(t)=-s(t) \psi(t)
$$

and

$$
\begin{gathered}
D q(t)=t \dot{q}(t)=2 \operatorname{Re}(t \dot{u}, u)=2 F \cdot e(A u, u)+\psi c_{1}=\ddot{c}\left(A_{1} u, u\right)+\psi q \\
D q(t)=\frac{t \dot{s} q+s t \dot{q}}{s q}=\frac{t \dot{q}-q}{q}=\frac{2}{q(t)}\left(A_{1} u, u\right) .
\end{gathered}
$$

Next it follows from (1.8) that $\ell(t)$ is twice differentiable, and

$$
\begin{aligned}
D \ell(t) & =\frac{2}{q} D\left(A_{1} u, u\right)-\frac{2}{a^{2}}\left(A_{1} u, u\right) D q \\
& =\frac{4}{q} \operatorname{Re}\left(A_{1} u, D u\right)-\frac{2}{q^{2}}\left(A_{1} u, u\right)\left[2\left(A_{1} u, u\right)+\psi q\right] \\
& =\frac{4}{q} \operatorname{Re}\left(A_{1} u, D u\right)-\frac{2 \psi}{q}\left(A_{1} u, u\right)-\frac{4}{q^{2}}\left(A_{1} u, u\right)^{2} \\
& =\frac{4}{q}\left(A_{1} u, A_{1} u\right)-\frac{2}{q^{2}}\left(A_{1} u, u\right)^{2}+\frac{4}{q} \operatorname{Re}\left(A_{1} u, f\right)-\frac{2 \psi}{q}\left(A_{1} u, u\right)+\frac{4}{q} \operatorname{Re}\left(A_{1} u, A_{2} u\right) .
\end{aligned}
$$

Now

and hence we find

$$
\frac{4}{q}\left[\left\|A_{1} u\right\|^{2}-q^{-1}\left(A_{1} u, u\right)^{2}\right]=\frac{4}{q}\left\|A_{1} u-\frac{\left(A_{1} u, u\right)}{q} u\right\|^{2},
$$

$$
D^{2} l(t)=\frac{4}{q}\left\|A_{1} u-\frac{\left(A_{1} u, u\right)}{q} u\right\|^{2}+\frac{4}{q} R e\left(A_{1} u, f\right)-\frac{2 \psi}{q}\left(A_{1} u, u\right)+\frac{4}{q} \operatorname{Re}\left(A_{1} u, A_{2} u\right) .
$$

From (1.4),

$$
\begin{aligned}
\frac{4}{q} \operatorname{Re}\left(A_{1} u, f\right)-\frac{2 \psi}{q}\left(A_{1} u, u\right) & =\frac{4}{q} \operatorname{Re}\left[\left(A_{1} u, f\right)-\frac{\left(A_{1} u, u\right)(u, f)}{q}\right] \\
& =\frac{4}{q} \operatorname{Re}\left(A_{1} u-\frac{\left(A_{1} u, u\right)}{q} u, f\right) \\
& \geq-\frac{2}{q}\left\|A_{1} u-\frac{\left(A_{1} u, u\right)}{q} u\right\|^{2}-\frac{4}{q}\|f\|^{2},
\end{aligned}
$$

and from (1.1)-(1.3),

$$
\|f(t)\| \leq t_{\phi}(t) q^{\frac{1}{2}}(t),
$$

so we have

$$
\begin{gathered}
D^{2} \ell(t) \geq \frac{2}{q}\left\|A_{1} u-\frac{\left(A_{1} u, u\right)}{q} u\right\|^{2}-2 t^{2} \phi^{2}(t)+\frac{4}{q} \operatorname{Re}\left(A_{1} u, A_{2} u\right) . \\
\operatorname{Re}\left(A_{1} u, A_{2} u\right)=\frac{1}{2}\left(\left[A_{1}, A_{2}\right] u, u\right)=0
\end{gathered}
$$

and hence we find

$$
D^{2} \ell(t)+2 t^{2} \phi^{2}(t) \geq 0
$$

Lemma 1.1 is proved. 
LEMMA 1.2. Let $\ell(\tau)$ the a solution of $\left(1.7^{\prime}\right)$. Then

$$
z(t) \geq \varepsilon\left(t_{0}\right)+\left[t_{0} \dot{i}\left(t_{0}\right)+4 c\right] \ln \frac{t}{t_{0}},
$$

where

PR.00F. Let

$$
c=\int_{0}^{t_{0}} t^{2}(t) d t
$$

Then we have

$$
\ell_{1}(t)=D \ell(t) \text {. }
$$

and

$$
D \ell_{1}(t)+4 t^{2} \phi^{2}(t)=f(t) \geq 0
$$

$$
\begin{aligned}
& \ell_{1}(t)=\ell_{1}\left(t_{0}\right)-4 \int_{t_{C}}^{t} \tau \phi^{2}(\tau) d \tau+\int_{t_{C}}^{t_{f(\tau)}} \frac{{ }^{2}}{\tau} d \tau,
\end{aligned}
$$

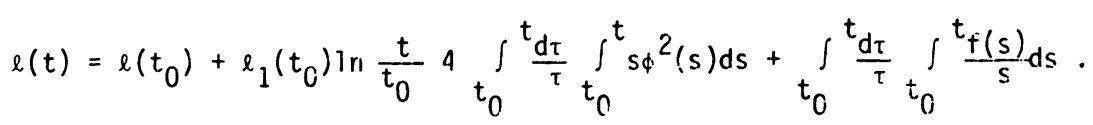

On condition that $t, \tau \leq t_{0}$, we have

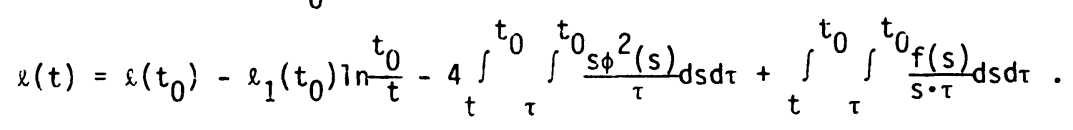

We assume that $f(s) \geq 0, s, \tau \in I$, i.e., $s \cdot \tau \geq 0$.

We have $\frac{f(s)}{s \cdot \tau} \geq 0$, and for $t \leq t_{0}, \tau \leq t_{0}$,

$$
\int_{t}^{t_{0}} \frac{d \tau}{\tau} \int_{t}^{t_{0}} \frac{f(s)}{s} d s \geq 0
$$

Next it follows from (1.10) and (1.11) that

$$
\begin{gathered}
\ell_{1}\left(t_{0}\right)=\left.t \dot{l}(t)\right|_{t=t_{0}}=\left.D l(t)\right|_{t=t_{0}}=t_{0} \dot{l}\left(t_{0}\right), \\
\int_{\tau}^{t_{0} 0} s \phi^{2}(s) d s \leq \int_{0}^{t_{0}} s \phi^{2}(s) d s=c
\end{gathered}
$$

and

$$
4 \int_{t}^{t_{0}} \frac{d \tau}{\tau} \int_{\tau}^{t_{0}} s \phi^{2}(s) d s \leq 4 c \int_{t}^{t_{0}} \frac{d \tau}{\tau}=4 c \ln _{t}^{t} 0
$$

Now we have

$$
\ell(t) \geq \ell\left(t_{0}\right)+\left[t_{0} \dot{i}\left(t_{0}\right)+4 c\right] \ln \frac{t}{t_{0}}
$$

and

$$
\begin{aligned}
& \exp (\ell(t)) \geq \exp \ell\left(t_{0}\right) \cdot \exp \left\{\left[t_{0} \dot{\ell}\left(t_{0}\right)+4 c\right] \ln \frac{t}{t_{0}}\right\} \\
& =\left.\operatorname{expl}\left(t_{0}\right) \cdot\left(t / t_{0}\right)^{t i(t)}\right|_{t=t_{0}} 4 c \cdot
\end{aligned}
$$

From the last formula,

$$
\exp \ell(t) \geq \exp \ell\left(t_{0}\right) \cdot\left(t / t_{0}\right)^{2 v+2 \mu},
$$


where

$$
\begin{gathered}
\nu=2 c \text { is dependent on } \phi(t) \text { on? } y^{\prime} \\
\mu=\frac{1}{2} \cdot t_{0} i\left(t_{0}\right) \text { is dependent on } \ell(t) \text { cnly. }
\end{gathered}
$$

l.emma 1.2 is proved.

We now turn to

PROOF OF THEOREM 1. From $(1.2)-(1.6)$,

$$
\begin{gathered}
q(t)=\exp \ell(t) / s(t), s\left(t_{0}\right)=1, q\left(t_{0}\right)=\exp \ell\left(t_{0}\right), \\
|\psi(\tau)| \leq 2 \tau \phi(\tau), 1 / s(t)=\exp \left(\int_{t_{0}}^{t} \frac{\psi(\tau)}{\tau}(\tau)\right.
\end{gathered}
$$

and

where

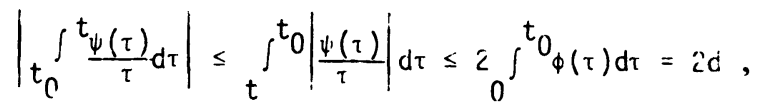

$$
d=\int_{0}^{t} 0 \phi(\tau) d \tau
$$

' and we have

$$
s^{-1}(t) \geq \exp (-2 d)
$$

and

$$
q(t) \geq e^{-2 d} \exp \ell(t) \geq \exp \ell\left(t_{0}\right) e^{-2 d}\left(t / t_{0}\right)^{2 \nu+2 \mu} .
$$

From (1.2) and (1.6) it follows that

$$
\|u(t)\| \geq M \| u\left(t_{0}\right)\left(t / t_{0}\right)^{\nu^{+} \mu},
$$

where $\nu, \mu$ are defined in (1.13) and (1.14), and $M=e^{-d}$

Assume that the flat-function $u(t)$ satisfying (1.1) is not identically zero. From (1.1i) we have that $u(t)$ is not a flat function. This is a contradiction. Therefore Theorem 1 is proved.

2. QUASIUNIQUENESS FOR PROBLEM (0.2).

THEOREM 2. Let $B(t)$ be a linear operator with domain $D_{B(t)}$. We shall assume that $u(t) \in D_{B}(t)$ and $B(t)=B_{1}(t)+B_{2}(t), B_{1}^{*}(t)=B_{1}(t), B_{2}^{*}(t)=-B_{2}(t)$ and $\operatorname{Re}(B(t) u, u)=\left(B_{1}(t) u, u\right), \operatorname{Re}\left(B_{2}(t) u, u\right)=0$, that $u(t) \varepsilon C^{1}(1, t), B(t) u(t) \varepsilon C^{1}(I, H)$, and $\phi(t)$ denotes a non-negative continuous function in the interval $I$.

We assume that the function $B(t) x$ is differentiable for $0 \leq t \leq T$ for all $x \in D_{B(t)}$, and set

Let $u(t)$ be a solution of

$$
\frac{d}{d t} B(t) x=B(t) x
$$

$$
\|D u(t)-B(t) u(t)\| \leq t_{\phi}(t)\|u(t)\| \text {. }
$$

such that

$$
\left\|t^{-1}\left[B_{1}, B_{2}\right] u+B_{1} u\right\| \leq \gamma(t)\left\|E_{1}(t) u(t)\right\|+\beta_{\beta}(t)\|u(t)\|, t \in I,
$$

or

$$
\frac{1}{t}\left(\left[B_{1}, B_{2}\right] u, u\right)+\left(B_{1}(t) u, u\right) \geq-\gamma(t) \mid\left(B_{j} u, u\right)-B(t)\|u(t)\|^{2}
$$

where $\gamma(t), \epsilon(t)$ are non-negative continuous functions in the interval $I$. If $u(t)$ is a flat-function, then $u(t) \equiv 0$ in $I$. 
PROOF OF THEOREM 2. Le.t

$$
\begin{gathered}
q(t)=(u(t), u(t)) \\
f(t)=\operatorname{tu}(t)-B(t) u(t)
\end{gathered}
$$

and let $\left(t_{1}, t_{0}\right]$ be a subinterval of $I$ such that $q(t)>0$ for $t_{1}<t \leq t_{0}$,

$$
\begin{aligned}
& \psi(t)=2 \operatorname{Pe}(f(t), u(t)) / q(t) \\
& s(t)=\exp \left\{-t_{0}^{s}{ }^{t} \frac{\psi(\tau)}{\tau} d_{\tau}\right\} \\
& p(t)=s(t) q(t) \\
& \varphi(t)=\log p(t) .
\end{aligned}
$$

LEMMA 2.1. Suppose that all the corditions of Theorem 2 hold. Then $\ell(t)$ is twice differentiable and satisfies the followirg second-nrder differential inecuality in the interval $\left(t_{1}, t_{0}\right]$ :

$$
D^{2} \ell(t)+2 t_{\gamma}(t)\left|D_{\ell}(t)\right|+2 t_{\beta}(t)+4 t^{2} \phi^{2}(t)+2 t^{2} \gamma^{2}(t) \geq 0 .
$$

PROOF OF LEMMA 2.1.

$$
\begin{aligned}
t \dot{q}(t)=2 \operatorname{Re}(t \dot{u}(t), u(t)) & =2 \operatorname{Re}(f, u)+2 \operatorname{Pe}(B(t) u, u) \\
& =\psi q+2\left(B_{1}(t) u, u\right) \\
t \dot{q}(t)=\frac{t \dot{p}(t)}{\dot{p}(t)}=\frac{t \dot{q} s+q t \dot{s}}{s q} & =\frac{2\left(B_{1}(t) u, u\right) s+\psi q s-\psi s q}{s q} \\
= & \frac{2}{q}\left(B_{1}(t) u, u\right)=\frac{2}{q}\left(b_{1} u, u\right) .
\end{aligned}
$$

Next it follows fron (2.11) that $\ell(t)$ is twice cifferentiable, and $D_{l}^{2}(t)=\frac{2}{q} D\left(B_{1} u, u\right)-\frac{2}{q^{2}}(B u, u) D q$

$$
\begin{aligned}
& =\frac{2}{q}\left[\left(D B_{1} u, u\right)+2 \operatorname{Re}\left(B_{1} u, D u\right)\right]-\frac{2}{a^{2}}\left(B_{1} u, u\right)\left[2\left(B_{1} u, u\right)+\psi q\right] \\
& =\frac{4}{q} \operatorname{Re}\left(B_{1} u, f\right)+\frac{4}{q}\left(B_{1} u, B_{2} u\right)+\frac{2}{q}\left(D B_{1} u, u\right)-\frac{4}{a^{2}}\left(B_{1} u, u\right)^{2}-\frac{2 \psi}{q}\left(B_{1} u, u\right)+\frac{4}{q} \operatorname{Re}\left(B_{1} u, B_{2} u\right)
\end{aligned}
$$

Now

$$
\frac{4}{q}\left[\left\|B_{1} u\right\|^{2}-q^{-1}\left(B_{1} u, u\right)^{2}\right]=\frac{4}{q}\left\|B_{1} u-\frac{\left(B_{1} u, u\right)}{q} u\right\|^{2}
$$

and

$$
D^{2} \ell(t)=\frac{4}{q}\left\|B_{1} u-\frac{\left(B_{1} u, u\right)}{q} u\right\|^{2}+\frac{4}{q} \operatorname{Re}\left(B_{1} u, f\right)+\frac{2}{q}\left(D B_{1} u, u\right)-\frac{2 \psi}{q}\left(B_{1} u, u\right)+\frac{4}{q} \operatorname{Re}\left(B_{1} u, B_{2} u\right) .
$$

From (2.6),

$$
\begin{aligned}
\frac{4}{q} \operatorname{Re}\left(B_{1} u, f\right)-\frac{2 \psi}{q}\left(B_{1} u, u\right) & =\frac{4}{q} \operatorname{Re}\left[\left(B_{1} u, f\right)-\left(\frac{\left(B_{1} u, u\right)}{q} u, f\right)\right] \\
& =\frac{4}{q} \operatorname{Re}\left(B_{1} u-\frac{\left(B_{1} u, u\right)}{q} u, f\right) \\
& \geq \frac{2}{q}\left\|B_{1} u-\frac{\left(B_{1} u, u\right)}{q} u\right\|^{2}-\frac{4}{q}\|f\|^{2} .
\end{aligned}
$$

(a) Case (2.3). From (2.3) we have

$$
\left(\left[B_{1}, B_{2}\right] u, u\right)+\left(D_{1} u, u\right) \geq-\gamma(t) t\left\|B_{1}(t) u(t)\right\| \cdot\|u(t)\| \cdot\|-B(t) \cdot t\| u(t) \|^{2}
$$

and

$$
D_{l}^{2}(t) \geq \frac{2}{q}\left\|B_{1} u-\frac{\left(B_{1} u, u\right)}{q} u\right\|^{2}-\frac{4}{q}\|f\|^{2}-\frac{2}{q}\left[\gamma(t) \cdot t \cdot B_{1} u\|\cdot\| u\left\|-B_{B}(t) \cdot\right\| u \|^{2}\right]
$$


or

$$
a^{2} \rho^{\prime}(t)+4 \phi^{2} t^{2}+2 i_{k}(t) \geq \frac{2}{q}\left\|B_{i} u-\frac{\left(E_{1}(1-u)\right.}{q} u\right\|^{2}-\frac{2 t_{y}(t)}{q(t)}\left\|B_{1} u\right\| \cdot\|u\|_{1} \text {. }
$$

For fixed $t$, we have

(i) $\left|\left(B_{1} u, u\right)\right| \geq \frac{1}{2}\left\|B_{1} u\right\| \cdot\|u\|^{\prime}$, or

(ii) $\left|\left(B_{1} u, i\right)\right|<\frac{1}{2}\left\|B_{1} u\right\|_{i} \cdot\|u\|_{i}$.

Ir. case $(i)$,

$$
\frac{2}{q}\left\|B_{1} u\right\| \cdot\|u\| \leq \frac{4\left|\left(B_{1} u, u\right)\right|}{q}=2|D e(t)|
$$

and

In case (ii),

$$
D^{2} \ell(t)+2 \operatorname{tr}(t)|D \ell(t)|+4 t^{2} \phi^{2}(t)+2 t_{\beta}(t) \geq 0
$$

$$
\left(B_{1} u, u\right)^{2}<\frac{1}{4}\left\|B_{1} u\right\|^{2}\|u\|^{2}=\frac{1}{4}\left\|^{\prime} B_{1} u\right\|^{2} q,
$$

and from the inequality

$$
\|a+b\|^{2} \geq \frac{1}{2}\|a\|^{2}-\|b\|^{2}
$$

it follows that

and

$$
\left\|B_{1} u-\frac{\left(B_{1} u, u\right)}{q} u\right\|^{2} \geq \frac{1}{2}\left\|B_{1} u\right\|^{2}-\frac{\left(B_{1} u, u\right)^{2}}{q^{2}} q \geq \frac{1}{4}\left\|B_{1} u\right\|^{2}
$$

and

$$
\begin{aligned}
D^{2} \ell(t)+4 \phi^{2} t^{2}+2 t B(t) & \geq \frac{1}{2 q}\left[\left\|B_{1} u\right\|^{2}-4 t_{\gamma}(t)\left\|B_{1} u\right\| \cdot\|u\|\right] \\
& =\frac{1}{2 q}\left[\left\|B_{1} u\right\|-2 t_{\gamma}\|u\|\right]^{2}-\frac{1}{2 q} 4 t^{2} \gamma^{2}(t)\|u\|^{2} \geq-2 t^{2}{ }^{2}(t),
\end{aligned}
$$

$$
D^{2} \ell(t)+4 t^{2} \phi^{2}+2 t^{2} \gamma^{2}+2 t \beta \geq 0 .
$$

(b) Case (2.3a). From (2.3a) we have

$$
\left(\left[B_{1}, B_{2}\right] u, u\right)+\left(D B_{1} u, u\right) \geq-t_{\gamma}(t)\left|\left(B_{1} u, u\right)\right|-t_{s}(t)_{q}(t)
$$

and

$$
D^{2} \ell(t) \geq \frac{2}{q}\left\|B_{1} u-\frac{\left(B_{1} u, u\right)}{q} u\right\|^{2}-\frac{4}{q}\|f\|^{2}-\frac{2}{q} t \gamma(t)\left|\left(B_{1} u, u\right)\right|-2 t_{B}(t) .
$$

From (2.5) we find

and from this and (2.11),

$$
\|f(t)\| \leq t \phi(t) q^{\frac{1}{2}}(t)
$$

$$
D^{2} \ell(t)+4 t^{2} \phi^{2}(t)+2 \operatorname{tr}(t)|D \ell(t)| \geq \frac{2}{q}\left\|B_{1} u-\frac{\left(B_{1} u, u\right)}{q} u\right\| \geq 0 .
$$

From (2.13)-(2.15) it follows that

where

$$
D^{2} \ell(t)+a(t)|D \ell(t)|+b(t) \geq 0
$$

Lenma 2.1 is proved.

$$
\begin{gathered}
a(t)=2 t \gamma(t) \\
b(t)=4 t^{2} \phi^{2}(t)+2 t^{2} \gamma^{2}(t)+2 t \beta(t) .
\end{gathered}
$$

LEMMA 2.2. Let $\ell(t)$ be a twice differentiable function in the intervar $l$, 
satisfying the following second-crder differential inequality

$$
\begin{gathered}
u^{\hat{c}} \ell(t)+t a ̈(t)|c l l(t)|+t b(t) \geq 0, t \in 1 \\
a(t) \leq M, t(t) \leq M, \forall t \in I
\end{gathered}
$$

where $a(t), b(t)$ are non-negative continuous functions in $I$. Then

$$
\ell(t) \geq \ell\left(t_{0}\right)+c_{1} \ln \frac{t}{t_{0}}+c_{2} \ln \frac{t}{t_{0}},
$$

where $c_{1}$ is a constant depending on $M, t_{0}, t$, and $c_{2}$ is a corstant deperiding only on $M$ and $t_{0}$. Hence,

$$
\exp l(t) \geq \exp l\left(t_{0}\right) \cdot t^{\nu} \cdot t^{\mu}
$$

with $\nu, \mu$ non-negative, $\nu$ a ccristant depending on $a$ and $b$ only, and $\mu$ a constant depending on $a, b$ and $t_{0} \dot{i}\left(t_{0}\right)$.

PROOF. From (2.16), it follows that

$$
D^{2} \ell(t)+M t|D \ell(t)|+M t \geq 0
$$

is true. We change the variable using the formula

$$
t=e^{-\tau}
$$

and for $\ell(\tau)$ we have

$$
\ell(\tau)+M e^{-\tau}|\dot{\ell}(\tau)|+M e^{-\tau} \geq 0 .
$$

From Lemma 1.2 of [2] we get

$$
\ell(\tau) \geq \ell\left(\tau_{0}\right)+\min \left\{0, \dot{\ell}\left(\tau_{0}\right)\right\} e^{\operatorname{Mi}_{i}{ }^{\tau} 0}\left(\tau-\tau_{0}\right)-M e e^{{ }^{\tau} 0} e^{\tau} 0\left(\tau-\tau_{0}\right) .
$$

From (2.20) and (2.21) we have

$$
\begin{aligned}
\ell(\tau) & \geq \ell\left(t_{0}\right)+\min \left\{0, t_{0} \dot{l}\left(t_{0} ;\right\} \exp \left(\frac{M}{t_{0}}\right) \ln \frac{t_{0}}{t}-M \exp \left(\frac{M}{t_{0}}\right) t_{0}^{-1} \ln \frac{t_{0}}{t}\right. \\
& =\ell\left(t_{0}\right)+\max \left\{0,-t_{0} \dot{l}\left(t_{0}\right)\right\} \exp \left(\frac{M}{t_{0}}\right) \ln \frac{t}{t_{0}}+M \exp \left(\frac{M}{t_{0}}\right) t_{0}^{-1} \ln \frac{t}{t_{0}} \\
& =\ell\left(t_{0}\right)+\mu\left(t_{0}\right) \ln \frac{t}{t_{0}}+v\left(t_{0}\right) \ln \frac{t}{t_{0}},
\end{aligned}
$$

where

$$
u\left(t_{0}\right)=\max \left\{0,-t i\left(t_{0}\right)\right\} \exp \left(M / t_{0}\right)
$$

depends only on $M, t_{0}, i\left(t_{0}\right)$, and

$$
v\left(t_{0}\right)=\operatorname{Mexp}\left(M / t_{0}\right) t_{0}^{-1},
$$

which depends on $M$ and $t_{0}$ only. From (2.22) we have

Lemma 2.2 is proved.

$$
e^{\ell(t)}=e^{\ell\left(t_{0}\right)} \cdot\left(t / t_{0}\right)^{\nu} \cdot\left(t / t_{0}\right)^{\mu} \text {. }
$$

REMARK. The theorem proved above corresponds to Theorem 1 of paper [1]. Our condition (2.3) exactly coincides with the condition (1.6) of [1]. Simultaneously the condition (2.3) is weaker than the corresponding condition (1.4) in [1]

Indeed, the condition (1.4) of [1] is of the form

$$
t^{-1}\left(\left[B_{1}, B_{2}\right] u, u\right)+\left(B_{1} u, u\right) \geq-\lambda\left(B_{1} u, u\right)-c(u, u)
$$

with $\lambda \geq 0, C \geq 0$. At the same time our condition (2.3) reads

$$
t^{-1}\left(\left[B_{1}, B_{2}\right] u, u\right)+\left(B_{1} u, u\right) \geq-\gamma(t)\left|\left(B_{1} u, u\right)\right|-B(t)(u, u),
$$

with $\gamma(t), B(t)$ non-negative continuous functions in the interval $I$. 


\section{MAIN THEORENS}

It car be easily seen from the proof of Theorem 2 for the case (2.3a) that the following is true:

THEOREM 3. Let $B(t)$ be a linear operaior with damain $D_{E}(t)$,

$$
B(t)=B_{1}(t)+B_{2}(t) \text {, }
$$

where

$$
\begin{aligned}
& B_{1}(t)=B_{1}^{\star}(t) \text { is the self-adjoint part of } B(t), \\
& B_{2}(t)=-B_{2}^{*}(t) \text { is the anti-self-acjoint part of } B(t) .
\end{aligned}
$$

We shail assume trat $u(t) \varepsilon D_{B}(t)$, that $u(t) \varepsilon C^{1}(I, H), B(t) u(t) \in C^{1}(I, H)$. Let $\phi(t)$ denote a non-negative continucus function ir the interval $I$. Let a flat-function $u(t)$ be a solution of

such that

$$
\|\sigma u(t)-B(t) u(t)\| \leq t_{\phi}(t)\|c u(t)\|
$$

$$
\begin{aligned}
\| B_{1} u & -\frac{\left(B_{1} u, u\right)}{(u, u)} u \|^{2}+\left(\left[B_{1}, B_{2}\right] u, u\right)+\left(\left(D_{1}\right) j u, u\right) \\
& \geq-r(t) t\left|\left(B_{1} u, u\right)\right|-B_{1}(t) t\|u(t)\|^{2},
\end{aligned}
$$

where $\gamma(t), \beta(t)$ are non-negative continuous functions in the interval $I$. Then $u(t) \equiv 0$ in $I$.

Now consider, instead of inequality (3.1), the equation

$$
t \frac{d u}{d t}=B(t) u(t) \text {, }
$$

with the same assumptions regarding $B(t)$ as in Theorem 3 . The following is true:

THEGREM 4. Let, with the assumptions of Theorem $3, u(t)$ be a flat-function and solution of the equation (3.3). Then, if

$$
\begin{gathered}
2\left\|B_{1} u-\frac{\left(B_{1} u, u\right)}{(u, u)} u\right\|^{2}+\left(\left[B_{1}, B_{2}\right] u, u\right)+\left(t B_{1} u, u\right) \\
\geq-\gamma(t) t\left|\left(B_{1} u, u\right)\right|-B(t) t\|u(t)\|^{2},
\end{gathered}
$$

then $u(t) \equiv 0$ in $I$.

PROOF. Let

$$
\begin{gathered}
q(t)=(u(t), u(t)), \\
\ell(t)=\log q(t)
\end{gathered}
$$

and

$$
\begin{aligned}
t \dot{q}(t)=2 \operatorname{Re}(t \dot{u}, u) & =2 \operatorname{Re}(B(t) u(t), u(t))=2\left(B_{1} u, u\right) \\
t \dot{l}(t) & =\frac{t \dot{q}(t)}{q(t)}=\frac{2}{q}\left(B_{1} u, u\right) .
\end{aligned}
$$

Next it follows from (3.7) that $\ell(t)$ is twice differentiable, and

$$
\begin{aligned}
D^{2} \ell(t) & =\frac{2}{q} D\left(B_{1} u, u\right)-\frac{2}{q^{2}}\left(B_{1} u, u\right) D q \\
& =\frac{2}{q}\left(t \hat{B}_{1} u, u\right)-\frac{4}{q} \operatorname{Re}\left(B_{1} u, D u\right)-\frac{2}{q^{2}}\left(B_{1} u, u\right) \cdot 2\left(B_{1} u, u\right) \\
& =\frac{2}{q}\left(t B_{1} u, u\right)-\frac{4}{q} \|\left(B_{1} u \|^{2}+\frac{4}{q} \operatorname{Re}\left(B_{1} u, B_{2} u\right)-\frac{4}{q^{2}}\left(B_{1} u, u\right)^{2}\right.
\end{aligned}
$$


Now

$$
\frac{4}{q}\left\|B B_{1} u\right\|^{2}-\frac{4}{q^{\prime}}\left(B_{1} u, u\right)^{2}=\frac{4}{q}\left\|B_{1} u-\frac{\left(B_{1} u, u\right)}{q} u\right\|^{2}
$$

and

$$
\begin{aligned}
\frac{4}{q} \operatorname{Re}\left(B_{1} u, D_{2} u\right) & =\frac{2}{q}\left[\left(B_{1} u, B_{c} u\right)+\left(B_{2} u, B_{1} u\right)\right] \\
& =\frac{2}{q}\left(\left(-B_{2} B_{1}+B_{1} B_{2}\right) u, u\right) \\
& =\frac{2}{q}\left(\left[B_{i} B_{2}\right] u, u\right)
\end{aligned}
$$

From this we find

$$
D^{2} \ell(t)=\frac{4}{q}\left\|B_{1} u-\frac{\left(B_{1} u, u\right)}{q}-u\right\|^{2}+\frac{2}{q}\left(\left[B_{1}, B_{2}\right] u, u\right)+\frac{2}{q}\left(t B_{1} u, u\right),
$$

and from (3.4) we have

$$
\begin{aligned}
D^{2}(t) & =\frac{2}{q}\left\{2\left\|E_{1} u-\frac{\left(E_{1} u, u\right)}{q} u\right\|^{2}+\frac{2}{q}\left(\left[B_{1}, E_{c} j u, u\right)+\frac{2}{q}\left(t B_{1} u, u\right)\right\},\right. \\
& \geq-\frac{2 t}{q}\left[\gamma(t)\left|\left(B_{1} u, u\right)\right|+B(t)\|u\|^{2},\right.
\end{aligned}
$$

and from (3.7) it follows that

$$
D^{2} \ell(t) \geq-\gamma(t) t|D \ell(t)|-\hat{c} t_{B}(t)
$$

or

$$
L^{2} \ell(t)+\gamma(t) t|D \ell(t)|+2 t \beta(t) \geq 0 .
$$

From (3.12) and Lemma 2.2 it follows immediately that $u(t) \equiv 0$ in the interval $I$. 4. REMARKS.

REMARK 1. Our key step in proving all the thecrems was to obtain an inequality for $\ell(t)$ of the form

$$
D^{2}(t)+t_{\alpha}(t)\left|D_{\ell}(t)\right|+t_{B}(t) \geq 0 \text {. }
$$

Therefore, it follows from (2.12) in the case of equation (3.3) (problem (0.1), i.e., when $f=\psi=0$ ), that the following equality holds:

$$
\begin{aligned}
D_{l}^{2}(t) & =\frac{4}{q}\left\|B_{1} u\right\|^{2}+\frac{2}{q}\left(t B_{1} u, u\right)-\frac{4}{q^{2}}\left(B_{1} u, u\right)^{2}+\frac{2}{q}\left(\left[B_{1}, B_{2}\right] u, u\right) \\
& =\frac{4}{q}\left\|B_{1} u-\frac{\left(B_{1} u, u\right)}{q} u\right\|^{2}+\frac{2}{q}\left(t B_{1} u, u\right)+\frac{2}{q}\left(\left[B_{1}, B_{2}\right] u, u\right) .
\end{aligned}
$$

In the course of deducing (4.1), one obtains the condition (3.4),

$$
2\left\|B_{1} u-\frac{\left(B_{1} u, u\right)}{q} u\right\|^{2}+\left(t B_{1} u, u\right)+\left(\left[B_{1}, B_{2}\right] u, u\right) \geq-t_{\gamma}(t)\left|B_{1} u, u\right|-t_{B}(t)\|u(t)\|^{2} \text {. }
$$

Let us point out that it seems to us that this condition, obtained from (4.2), must be close enough to being necessary (for quasiuniqueness).

REMARK 2. For $t=0,(3.4)$ reduces to

$$
2\left\|B_{1} u-\frac{\left(B_{1} u, u\right)}{q} u\right\|^{2}+\left.\left(\left[B_{1}, B_{2}\right] u, u\right)\right|_{t=0} \geq 0,
$$

and since

$$
B_{1}=\frac{B+B^{*}}{2}, B_{2}=\frac{B-B^{*}}{2},
$$

it follows that

$$
\begin{gathered}
\left(B_{1} u, u\right)^{2}=\frac{1}{4}\left\{(B u, u)^{2}+|(B u, u)|^{2}+\left(B^{*} u, u\right)^{2}+\left|\left(B^{*} u, u\right)\right|^{2}\right\}, \\
2 \operatorname{Re}\left(B_{1} u, B_{2} u\right)=\left(\left[B_{1}, B_{2}\right] u, u\right)=\frac{1}{2}\left\{\|B u\|^{2}-\left\|B^{*} u\right\|^{2}\right\},
\end{gathered}
$$


and

$$
\left\|B_{1} u\right\|^{2}=\frac{1}{4}\left\{\|B\|^{2}+\left\|B^{*} u\right\|^{2}\right\}+\frac{1}{2} P e\left(B u, B^{*} u\right) .
$$

In this case our cordition reduces to the following ore:

$$
\begin{aligned}
& \frac{1}{2}|B u|^{2}+\frac{1}{2} \mid B^{\star} u \|^{2}+\operatorname{Re}\left(B u, B^{\star} u\right)-\frac{1}{2 q}\left\{(B u, u)^{2}+|(B u, u)|^{2}\right\} \\
& \left.-\left.\frac{1}{2 q^{2}}\left\{\left(B^{\star} u, u\right)^{2}+\mid B^{\star} u, u\right)\right|^{2}\right\}+\frac{1}{2}\|B u\|^{2}-\frac{1}{2}\left\|B^{\star} u\right\|^{2} \mid t=0 \geq 0 .
\end{aligned}
$$

For this condition to hold it is sufficient to have

$$
\|R u\|^{2}-\left\|B^{*} u\right\|^{2} \mid t=0 \geq 0
$$

or

$$
\left.\left[B_{1}, B_{2}\right]\right|_{t=0} \geq 0 \text {, }
$$

i.e., if $u$ is a flat solution of $(0.2)$ and (4.4) holds, ther $u \equiv 0$ in $I$ (see. also Remark 6).

REMARK 3. The method of the proof of the theorems concerning the quasiuniqueness of the solution of $(0.1)-(0.2)$ presented in sections 2 anc 3 allows one to assert, even in cases when there is no quasiuniqueness, that a given solution is trivial if the appropriate conditions are true for this solution. We have in mind the ccriditions $(2.3),(2.3 \mathrm{a}),(3.2),(3.4)$.

It may quite happen that these conditions do not hold for all the solutions of $(0.1)-(0.2)$. On the other hand, if for some specific solution $u(t)$ of $(0.1)$ or (0.2) the appropriate condition does hold, then its triviality follows from the flatriess of this specific $u(t)$. Quasiuniqueness of solution of $(0.1)-(0.2)$ follows in the case when these conditions are satisfied by the whole class of possibie solutions.

PEMARK 4. It follows from Theorems 3 and 4 of section 3 that the quasiuniqueness takes place:

(i) If $B$ does not depend on $t$, and $B_{2}(t)=0$, i.e., for any constant symmetric operator $B$.

(ii) If $B_{2}(t)=0$ and $B_{1}(t)$ sattisfies the condition

$$
c\left\|B_{1} u-\frac{\left(B_{1} u, u\right)}{(u, u)} u\right\|^{2}+\left(t B_{i_{t}} u, u\right) \geq-\gamma(t) t\left|\left(B_{1} u, u\right)\right|-t B(t)\|u(t)\|^{2} \text {. }
$$

Here $B_{1}(t)$ can be replaced by $B(t)$ and $C=1$ for the problen (0.2) and $C=2$ for the problem $(0.1)$ correspondingly.

(iii) If $B_{1}(t)=0$.

On the other hand, in case ( $i i i)$ there even exists a classical uniqueness in the case of $(0.1)$. This stems from the following:

$$
\begin{gathered}
t \frac{d u}{d t}=B(t), \\
\left(t \frac{d u}{d t}, u(t)\right)=\operatorname{Re}(B(t) u, u)=0, \\
\frac{1}{2} t \frac{d}{d t}(u, u)=\frac{1}{2} t \frac{d g}{d t}=0,
\end{gathered}
$$

and if $q(0)=0$, then $q(t)=0$ for all $t$.

REMARK 5. The conditions in Theorems 2-4 do not seem natural, at any rate not at first sight. The following conditions seem more natural:

(i) $\left(B_{1} u, u\right) \geq-\gamma(t)\left\{\left|\left(B_{1} u, u\right)\right|+\|u\|^{2}\right\}$ with $\gamma(t)$ a continuous function in $I$. (ii) $\operatorname{Re}\left(B_{1} u, B_{2} u\right)=\frac{1}{2}\left(\left[B_{1}, B_{2}\right] u, u\right) \geq-r(t)\left\{\left|\left(B_{1} u, u\right)\right|+\|u\|^{2}\right\}$ with the same $r(t)$. Then we have, from $(2.12)$, 


$$
\begin{aligned}
D^{2} \sim(t)+\left.\frac{4}{q}\right|^{\prime} f \|^{2} & \left.\geq \frac{2}{q} \mid\left\|B_{1} u-\frac{(B, y, u)}{q} u\right\|^{2}+\left(t B_{1} u, u\right)+\left(\left[B_{1}, B_{2}\right] u, u\right)\right\} \\
& \left.\geq-\frac{2}{q} \gamma(t) t\left\{\left|\left(B_{1} u, u\right)\right|+\|u\|^{2}\right\}-\frac{2}{q} 2 \gamma(t)\left\{\mid B_{1} u, u\right) \mid+\|u\|^{2}\right\}
\end{aligned}
$$

or

$$
0^{2} u(t)+4 t^{2}{ }_{\phi}^{2}(t)+\left[2 \gamma(t)+t_{\gamma}(t)\right]|D e(t)|+2\left[2 \gamma(t)+t_{\gamma}(t)\right]\|u\|^{2} \geq 0
$$

or, introducing a new function

$$
R(t)=2 \gamma(t)+t_{\gamma}(t)
$$

one obtains for $\ell(t)$,

$$
D^{2} \ell(t)+\beta(t)\left|D_{\ell}(t)\right|+{ }^{2} \beta(t)+4 t^{2} \phi^{2}(t) \geq C
$$

or

$$
2 \ell(t)+\beta(t)|D \ell(t)|+\alpha(t) \geq 0 .
$$

One can show, using the above reasoning, that

(a) in the case $\gamma(t)=t^{\varepsilon} \gamma_{1}(t), \varepsilon>0$, with $\gamma_{1}(t)$ bourded in $I$, there is quàsiuniqueness,

(b) in the case $\gamma(t)=M+t^{\varepsilon} \gamma_{1}(t), M>0, \varepsilon>0$, with $\gamma_{1}(t)$ bounded in $I$, the following estimate can be obtâined:

$$
\|u(t)\|^{2} \geq c t\left\|u\left(t_{0}\right)\right\|^{2} \exp \left[-(\nu+\mu)\left(t_{c} / t\right)^{2 k_{i}}\right]
$$

where

$$
\nu-\min \frac{a(\tau)}{\beta^{2}(t)}=\frac{1}{2 H}, \quad \mu=\min \left\{0, t_{0} \dot{\ell}\left(t_{0}\right)\right\} \frac{1}{2 M} .
$$

Let us point cut that in (4.8) we have a flat function.

(c) in the case of $\forall \varepsilon>0, \exists \delta>0: \forall t \quad[0, \delta] \gamma(t)<\varepsilon$.

Then the following estimate can be obtained:

$$
\|u(t)\|^{2} \geq c t\left\|u\left(t_{0}\right)\right\|^{2} \exp \left[-(\nu+\mu)\left(t_{0} / t\right)^{2 \varepsilon}\right], \forall \varepsilon>0
$$

where

$$
\nu=\frac{1}{2 \varepsilon}, \mu=\min \left\{0, t_{0} \dot{l}\left(t_{0}\right)\right\} \frac{1}{2 \varepsilon}, t_{0} \varepsilon[0, \delta] .
$$

We point out that in this case we have a flat function in (4.9).

REMARK 6 . Consider the two following terms with our conditions:

$$
\left\|B_{1} u-\frac{\left(B_{1} u, u\right)}{(u, u)} u\right\|^{2}+\left(\left[B_{1}, B_{2}\right] u, u\right) .
$$

We may have that the first non-negative term may improve the possible negativity of the second one. Unfortunately this is not the case. Let $\left\{e_{k}\right\}$ be an orthonormal basis of the eigenvectors of the operator $B_{1}$, assumed independent of $t$. Assume that the expression (4.10) is non-negative. Since the first term is identically zero on $\left\{e_{k}\right\}$, we then have

$$
\left(\left[B_{1}, B_{2}\right] \epsilon_{k}, e_{k}\right) \geq 0 \text {. }
$$

Taking into account the orthogonality of $\left\{e_{k}\right\}$ and (4.11), we obtain the following:

$$
\left(\left[\mathrm{B}_{1}, \mathrm{~B}_{2}\right] u, u\right) \geq 0 \text {. }
$$

i.e., the appropriate term is non-negative.

5. EXAMPLE.

Let us consider the following equation

$$
t \frac{\partial u}{\partial t}=\frac{\partial}{\partial x}\left[-k_{1}(x) \frac{\partial u}{\partial x}\right]+t \frac{\partial}{\partial x}\left[-k_{2}(x) \frac{\partial u}{\partial x}\right]=A(t) u
$$


where $x \in \Omega=\left[-1,1 j, t \in(0,1]\right.$. Here $H$ is Hilbert space $L_{2}(\Omega)$ with coridition

$$
\left.u\right|_{\partial \Omega}=0
$$

and standard scalar product.

$k_{1}(x), K_{2}(x)$ are smooth encugh (from $\left.c^{2}\right)$ real-valued functions. In this case $A(t)$ will be the self-adjoint operator on $D_{A(t)} \subseteq H$ ard

$$
\begin{gathered}
(A(t) u, u)=\left(k_{1}(x) \frac{\partial u}{\partial u}, \frac{\partial u}{\partial u}\right)+t\left(k_{2}(x) \frac{\partial u}{\partial u}, \frac{\partial u}{\partial u}\right) \\
(A(t) u, u)=\left(k_{2}(x) \frac{\partial u}{\partial u}, \frac{\partial u}{\partial u} ; .\right.
\end{gathered}
$$

Let us consider the following $k_{1}(x)$ and $k_{2}(x)$

$$
\begin{aligned}
& k_{1}(x)= \begin{cases}x^{3} & x \leq 0 \\
0 & x>0\end{cases} \\
& k_{2}(x)= \begin{cases}x^{3} & x \leq 0 \\
x^{4} & x>0\end{cases}
\end{aligned}
$$

Then from $(5.3)-(5.4)$ we have

$$
(A(t) u, u)=\left.(1+t)\left(x^{3} \frac{\partial u}{\partial x}, \frac{\partial u}{\partial x}\right)\right|_{\text {on }}(-1,0)+\left.t\left(x^{4} \frac{\partial u}{\partial x}, \frac{\partial u}{\partial x}\right)\right|_{\text {on }}(c,+1)
$$

and

$$
(A(t) u, u)=\left.\left(x^{3} \frac{\partial u}{\partial x}, \frac{\partial u}{\partial x}\right)\right|_{\text {on }(-1,0)}+\left.t\left(x^{4} \frac{\partial u}{\partial x}, \frac{\partial u}{\partial x}\right)\right|_{\text {on }}(0,+1)
$$

Theorem 1 of [1] does not work in this case:

i) $h(t)$ is not regative;

ii) $([\lambda A(t)+A(t)] u, u)$ is not positive for all $\lambda>0$;

iii) there is not an estimate of type $\left\|A_{1}(t) u\right\| \leq C(\|A u\|+\|u\|)$ for any $C>0$.

But from (5.7)-(5.8) we have that

$$
(A(t) u, u) \geq-2(A(t) u, u)
$$

and from Theorem 2, we obtain that the quasiuniqueness takes place for equation (5.1) under our assumptions (5.5)-(5.6).

REMARK. Of course, it is possible to construct an example of this

type with $C^{\infty}$ ccefficients. It is possible also to construct an example of this type for non-self-adjoint operator $A(t)$.

ACKNOWLEDGEMENT. I wish to thank Professor $Y$. Kannai for the very fruitful discussions and guidance.

\section{REFERENCES}

1. ALINHAC, S. and BAOUENDI, M. S. Uniqueness for the Characteristic Cauchy Problem and Strong Unique Continuation for Higher Order Partial Differential Inequalities, Amer. J. of Math., $102(1980), 179-217$.

2. BAOUENDI, M. S. and GAOULAOUIC, C. Cauchy Problem with Characteristic Initial Hypersurface, Comm. Pure Appl. Math., XXVI (1973), 445-475.

3. BAOUENDI, M. S. and GAOULAOUIC, C. Cauchy Problems with Multiple Characteristics in Spaces of Regular Distributions, Russian Math. Surveys, 29 (1974), $72-78$.

4. BAOUENDI, M. S., GAOULAOUIC, C. arid LIPKIN, L. J. On the Operator $\Delta r^{2}+\mu(\partial / \partial r) r+\S$, Diff. Equations, $15(1974), 499-509$.

5. BAOUENDI, M. S. and SJOSTRAND, J. Regularite Analytique pour des Operateurs Elliptiques Singulie:s en on Point, Arkiv fur Math., 14 (1976), 9-33. 
6. SCHUCHMAN, $v$. On the Quasiuniqueness of Solutions of Degenerate Elitiptic Equations, subnitted.

7. AGNON, S. and NIRENBERG, L. Properties of Solutions of Ordinary Differential Equations in Barach, Space, Cunn. Pure Appl. Math., 16 (1963), 121-239.

8. AGMON, S. Unicite et Convexite dans les Problemes Differentieles, Montrea?. 1966. 


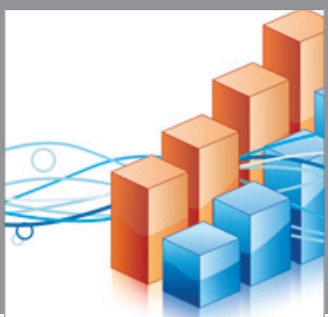

Advances in

Operations Research

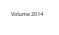

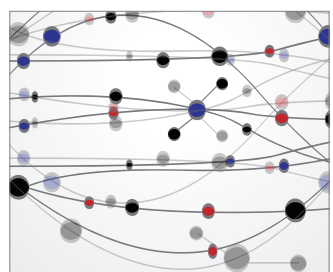

\section{The Scientific} World Journal
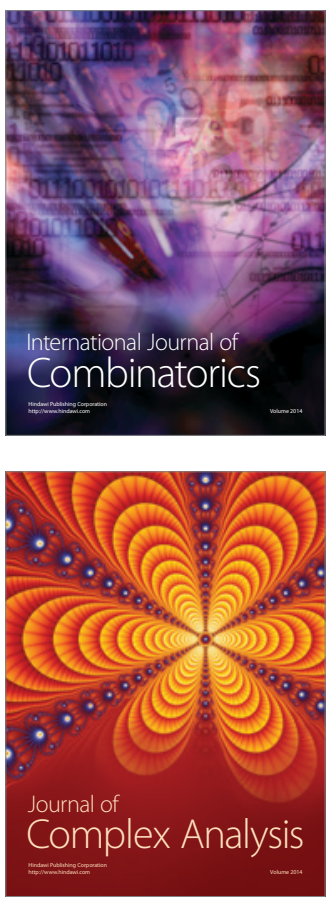

International Journal of

Mathematics and

Mathematical

Sciences
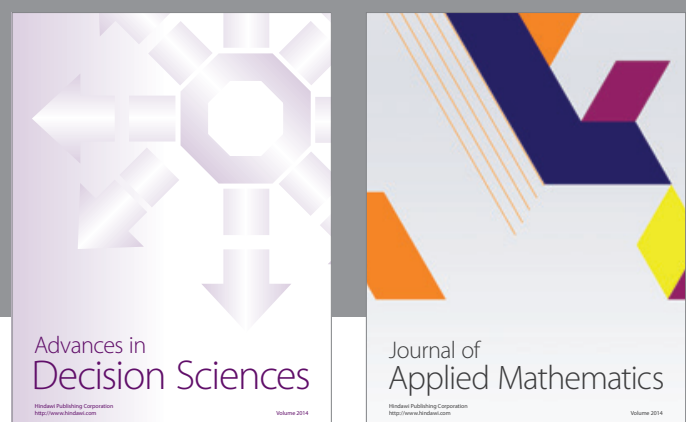

Journal of

Applied Mathematics
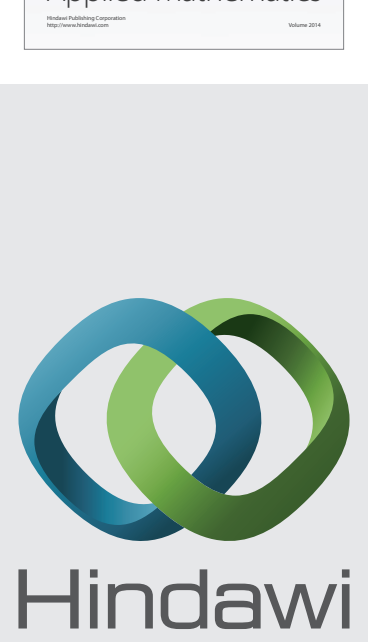

Submit your manuscripts at http://www.hindawi.com
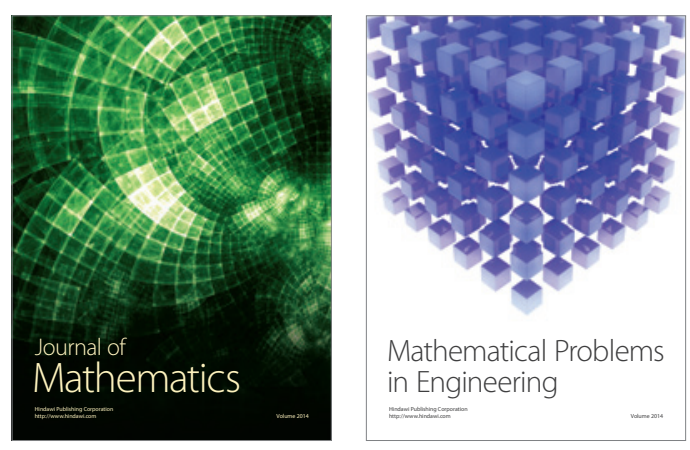

Mathematical Problems in Engineering
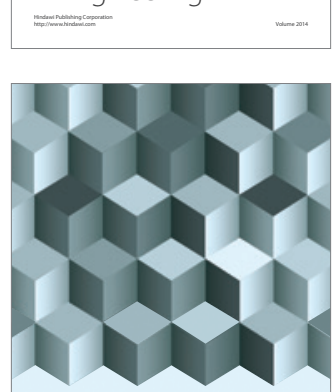

Journal of

Function Spaces
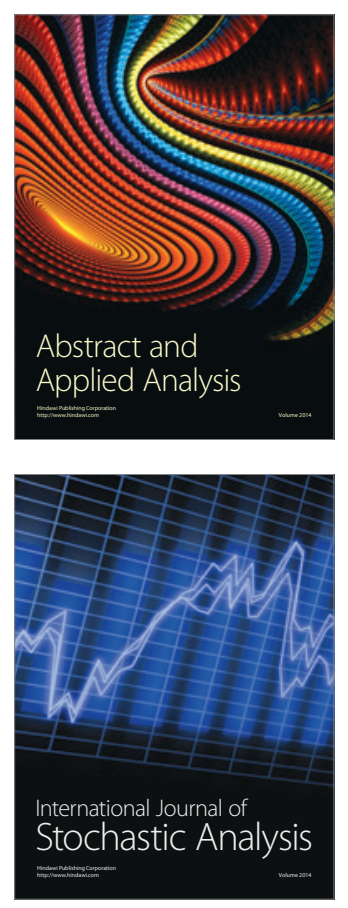

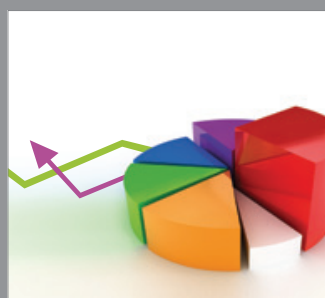

ournal of

Probability and Statistics

Promensencen
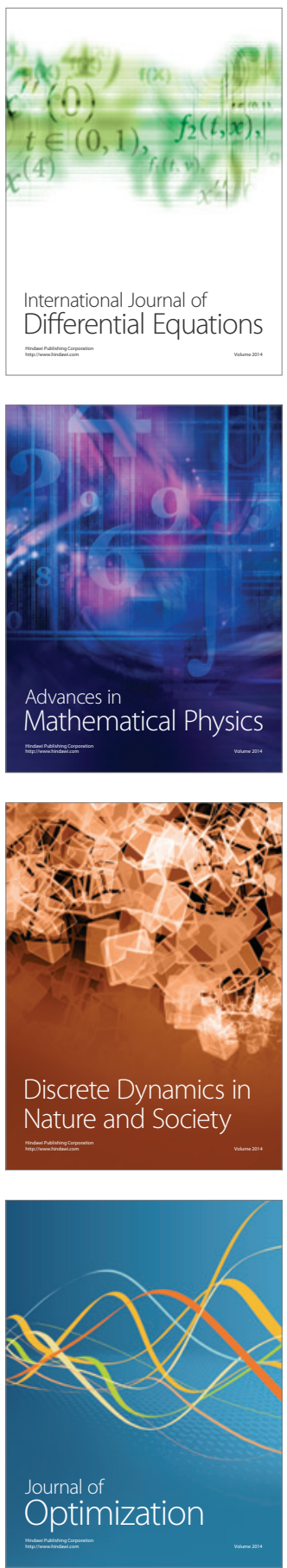